CORRECTION

\title{
Correction: A Large Collection of Novel Nematode-Infecting Microsporidia and Their Diverse Interactions with Caenorhabditis elegans and Other Related Nematodes
}

\section{The PLOS Pathogens Staff}

In Table 2, dividing lines were introduced during typesetting that affect the readability of the table. The publisher apologizes for the error. Please see the corrected Table 2 here.

Citation: The PLOS Pathogens Staff (2017)

Correction: A Large Collection of Novel NematodeInfecting Microsporidia and Their Diverse Interactions with Caenorhabditis elegans and Other Related Nematodes. PLoS Pathog 13(2): e1006204. doi:10.1371/journal.ppat.1006204

Published: February 15, 2017

Copyright: ๑ 2017 The PLOS Pathogens Staff. This is an open access article distributed under the terms of the Creative Commons Attribution License, which permits unrestricted use, distribution, and reproduction in any medium, provided the original author and source are credited. 
Table 2. Collection of other microsporidia species infecting wild nematodes

\begin{tabular}{|c|c|c|c|c|c|}
\hline Microsporidia & Nematode host & Strain & Country / Year & Sample composition & Spore feature \\
\hline \multirow{3}{*}{$\begin{array}{l}\text { Nematocida } \\
\text { majorn. sp. }\end{array}$} & C. briggsae & JUm2507 & Thailand / 2013 & rotting fruit & $2 S$ \\
\hline & C. briggsae & JUm2747 & Guadeloupe / 2014 & rotting fruit & $2 S$ \\
\hline & C. tropicalis & JUm2751 & Guadeloupe / 2014 & rotting fruit & n. d. \\
\hline \multirow{2}{*}{$\begin{array}{l}\text { Nematocida } \\
\text { minorn. sp. }\end{array}$} & Oscheius tipulae & JUm1510 & Czech Republic / 2008 & rotting apple & $2 S$ \\
\hline & O. tipulae & JUm2772 & Armenia / 2014 & rotting fruit & n. d. \\
\hline \multirow{2}{*}{$\begin{array}{l}\text { Nematocida } \\
\text { homosporus } \mathrm{n} . \mathrm{sp} .\end{array}$} & O. tipulae & JUm1504 & France / 2008 & rotting stem & $1 \mathrm{~S}$ \\
\hline & Rhabditella typhae & NICm516 & Portugal / 2013 & rotting apple & $1 S$ \\
\hline $\begin{array}{l}\text { Nematocida } \\
\text { ciargin. sp. }\end{array}$ & Procephalobus sp. & JUm2895 & Spain / 2015 & rotting fruit & $1 S$ \\
\hline $\begin{array}{l}\text { Nematocida } \\
\text { sp. } 7\end{array}$ & C. sp. 42 & NICm1041 & French Guiana / 2014 & flower & n. d. \\
\hline $\begin{array}{l}\text { Enteropsectra } \\
\text { Ionga n. sp. }\end{array}$ & Oscheius sp. 3 & JUm408 & Iceland / 2002 & compost & 1S, LT, AP \\
\hline \multirow{3}{*}{$\begin{array}{l}\text { Enteropsectra } \\
\text { breven. sp. }\end{array}$} & O. tipulae & JUm2551 & France / 2013 & rotting apple & 1S, SR, AP \\
\hline & O. tipulae & JUm1483 & France / 2008 & rotting plum & 1S, SR,AP \\
\hline & O. tipulae & JUm1456 & France / 2008 & rotting fruit & n. d. \\
\hline \multirow{4}{*}{$\begin{array}{l}\text { Pancytospora } \\
\text { philotis } \mathrm{n} . \mathrm{sp} \text {. }\end{array}$} & O. tipulae & JUm1505 & France / 2008 & rotting apple & LT \\
\hline & O. tipulae & JUm1460 & France / 2008 & rotting snail & LT \\
\hline & O. tipulae & JUm1670 & France / 2009 & rotting apple & LT \\
\hline & O. tipulae & JUm2552 & France / 2013 & rotting stem & LT \\
\hline $\begin{array}{l}\text { Pancytospora } \\
\text { epiphaga n. sp. }\end{array}$ & C. brenneri & JUm1396 & Colombia / 2008 & rotting fruit & LT \\
\hline
\end{tabular}

The reference strain of each newly found species is in bold. 2S: two distinct sizes of spores; 1S: one size of spores

LT: long, thin rod; SR: small rod (see dimensions in Table 3); AP: form spores first along the apical side of the intestinal cells. n. d.: not determined

doi:10.1371/journal.ppat.1006204.t001

\section{Reference}

1. Zhang G, Sachse M, Prevost M-C, Luallen RJ, Troemel ER, Félix M-A (2016) A Large Collection of Novel Nematode-Infecting Microsporidia and Their Diverse Interactions with Caenorhabditis elegans and Other Related Nematodes. PLoS Pathog 12(12): e1006093. doi:10.1371/journal.ppat.1006093 PMID: 27942022 